\title{
Polymorphism in Murine mtATP8 Gene Correlates with Decreased Reactive Oxygen Species in Aging Hematopoietic Cells
}

\author{
CATRIN ROOLF ${ }^{1}$, CHRISTIN KRETZSCHMAR ${ }^{1 *}$, KATRIN TIMMER $^{1}$, ANETT SEKORA $^{1}$, GUDRUN KNÜBEL $^{1}$, \\ HUGO MURUA ESCOBAR ${ }^{1}$, GEORG FUELLEN ${ }^{2}$, SALEH M. IBRAHIM ${ }^{3}$, MARKUS TIEDGE ${ }^{4}$, \\ SIMONE BALTRUSCH ${ }^{4}$, SARAH MÜLLER ${ }^{5}$, RÜDIGER KÖHLING ${ }^{6}$ and CHRISTIAN JUNGHANSS ${ }^{1}$ \\ Departments of ${ }^{1}$ Medicine III, Hematology/Oncology/Palliative Medicine and \\ ${ }^{5}$ Medicine II, Division of Gastroenterology, Rostock University Medical Center, Rostock, Germany; \\ Institutes of ${ }^{2}$ Biostatistics and Informatics in Medicine and Ageing Research and \\ ${ }^{4}$ Medical Biochemistry and Molecular Biology, Rostock University Medical Center, Rostock, Germany; \\ ${ }^{3}$ Institute of Experimental Dermatology, University of Lübeck, Lübeck, Germany; \\ ${ }^{6}$ Oscar Langendorff Institute of Physiology, Rostock University Medical Center, Rostock, Germany
}

\begin{abstract}
Background: Mitochondrial DNA (mtDNA) encodes for the respiratory chain proteins. Genetic alterations in mtDNA have been described during aging and linked to impaired hematopoiesis. Materials and Methods: We investigated two novel conplastic mouse strains harboring a mitochondrial nt7778 G/T polymorphism leading to an amino acid exchange in respiratory chain complex $V$. Effects on reactive oxygen species (ROS) and adenosine triphosphate (ATP) levels, as well as bone marrow composition and peripheral blood counts, were investigated during aging (up to 24 month). Results: The polymorphism correlated with significantly decreased ROS levels in aged mice. Effects on hematopoiesis were marginal and not statistically significant: numbers of erythroid cells in bone marrow, as well as mean corpuscular hemoglobin, tended to decrease over time. Conclusion: The investigated polymorphism is associated with decreased ROS levels in aged hematopoietic cells but does not significantly influence hematopoiesis itself.
\end{abstract}

This article is freely accessible online.

*These Authors contributed equally to this work.

Correspondence to: Christian Junghanss, Department of Medicine III, Hematology/Oncology/Palliative Medicine, Rostock University Medical Center, Ernst-Heydemann-Str. 6, 18057 Rostock, Germany. Tel: +493814947420, e-mail: Christian.Junghanss@med.unirostock.de

Key Words: mtDNA, stem cells, ROS, conplastic mice, hematopoiesis.
Aging of the hematopoietic system is accompanied by several changes in different blood compartments. For example, an immune shift in aged individuals has been described, leading to a higher percentage of myeloid cells with a concomitant enhanced susceptibility to infections and development of anemia $(1,2)$. Clinically, elderly individuals are more affected by myelodysplastic syndromes (MDS) and leukemia (3).

At the subcellular level, several modifications have been described during aging in human hematopoietic stem cells (HSCs). Elevated levels of phoshorylated histone H2AX (gammaH2AX), correlating with DNA damage, were found in HSCs of older individuals in comparison to HSCs of the umbilical cord blood (4). A transgenic mouse model of MDS showed increased levels of reactive oxygen species (ROS), correlating with increased levels of DNA damage and a more intensive progression from MDS to acute myelogenous leukemia (5). Further mouse models investigated the influence of manipulated mitochondrial DNA (mtDNA) on hematopoiesis. They revealed a correlation between impaired mtDNA function, elevated ROS levels and deregulated HSC function (6-8).

The mtDNA encodes for 13 proteins that are all part of the respiratory chain complexes (9). The respiratory chain generates a proton gradient (complex I, III and IV), allowing the respiratory chain complex $\mathrm{V}\left(\mathrm{F}_{\mathrm{O}} \mathrm{F}_{1}\right.$-ATPase $)$ to synthesize adenosine triphosphate (ATP). Besides ATP synthesis, the complexes of the respiratory chain are also the main source for ROS in the cell. ROS molecules as superoxide or hydrogen peroxide $\left(\mathrm{H}_{2} \mathrm{O}_{2}\right)$ are highly reactive and, at high concentrations, able to cause serious damage to cell components, such as double-strand breaks (10) and 
membrane disintegration $(11,12)$. Moreover, it was shown that elevated ROS levels lead to reduced maintenance and repopulation capacity of murine hematopoietic stem cells (HSC) (13).

Nevertheless, $\mathrm{H}_{2} \mathrm{O}_{2}$ carries out important positive functions in the cell as increased scavenging of ROS by overexpression of antioxidants leads to decreased proliferation by inactivation of Akt (14). Several signaling pathways are described to be activated by ROS generation, including NF-kB, PI3K, MAPK and p53 (15). Furthermore, $\mathrm{H}_{2} \mathrm{O}_{2}$ stimulation induces proliferation of mammalian cells $(14,16,17)$. It was also shown that signals stimulating hematopoietic growth factors are transduced by ROS formation (18). However, the link between ROS, mtDNA mutations, hematopoietic aging and hematological diseases are not yet completely understood.

Here, two conplastic mouse strains differing in one single polymorphism within the mtDNA ATP8 gene were comparatively analyzed. In the respiratory complex $\mathrm{V}$, the ATP8 protein is part of the proton channeling $\mathrm{F}_{\mathrm{O}}$ subunit. The C57BL/6-mt $\mathrm{t}^{\mathrm{FVB} / \mathrm{NJ}}$ (mtFVB) strain harbors the nt7778G/T polymorphism, while the control C57BL/6$\mathrm{mt}^{\mathrm{AKR} / \mathrm{J}}$ (mtAKR) strain is unaltered at this position. The replacement of aspartic acid, which lacks an aromatic group with tyrosine at the fifth position of the highly-conserved $\mathrm{N}$ terminal region, is important for proper complex $\mathrm{V}$ assembly (19). Due to the proton channeling function of subunit $\mathrm{F}_{\mathrm{O}}$, we hypothesize that the polymorphism will alter the transport of protons by complex $\mathrm{V}$ and, therefore, the generation of the proton gradient. It would, thus, impact the whole respiratory chain, influencing ROS and ATP production. Recently, the impact of the nt7778 G/T polymorphism on cell function has been studied in different tissues (19-22), as well as its effects on anxiety and stress reactivity $(23,24)$.

We analyzed the effects of this polymorphism on the hematopoietic system, particularly in hematopoietic stem cell function during aging. Groups of both strains at three different time points ranging from young (3 months), midaged (12 months) to advanced-aged (24 months) were included in the study. In addition to analyzing the blood count at these time points, we also investigated the ROS and ATP levels of bone marrow cells (BMC), as well as the proportion of distinct subpopulations. Furthermore, an in vivo study was performed to investigate the restoration of hematopoiesis after cytostatic stress. This revealed a correlation between the nt7778 G/T polymorphism and the aging process in murine hematopoiesis.

\section{Materials and Methods}

Animals. Conplastic mouse strains were implemented as described before (24). In brief, due to the maternal inheritance of mtDNA, female animals of the mitochondrial donor strain AKR/J and $\mathrm{FVB} / \mathrm{NJ}$, respectively, were crossed with males with the corresponding genomic background (C57BL/6NTac). Female offspring was then bred with males of the background strain. After ten generations the conplastic mouse strain harbored a stable mtDNA of the donor strain, as well as a stable nucleic DNA of the background strain. Animals were bred and housed under specific pathogen-free conditions in the central animal facility of the University of Rostock, Germany. Animals were kept under a 12-hour light/dark cycle and had access to water and standard laboratory chow ad libitum. All animals received human care according to the German legislation on protection of animals and the Guide for the Care and Use of Laboratory Animals (NIH publication 86-23, revised 1985); all efforts were made to minimize suffering. For in vitro analysis of BMC, male and female animals at 3,12 and 24 months of age were investigated. For in vivo study, animals at 3 and 12 months were examined. Animal experiments were approved by the state's Animal Care Committee of Mecklenburg-Western Pomerania (LALLF M V/TSD/7221.3-1.1-100/12).

Isolation of BMC. Animals were sacrificed under narcosis (ketamine $65 \mathrm{mg} / \mathrm{kg}$ of body weight and xylazine $13 \mathrm{mg} / \mathrm{kg}$ of body weight) by cervical dislocation. Tissue was removed from femora and tibiae, which were cut at both sides and flushed several times with PBS. Cells were collected in falcon tubes and kept on ice until further processed.

Blood count. Blood samples were obtained from the retro-orbital plexus using an ethylenediaminetetraacetic acid (EDTA)-coated capillary and collected in EDTA-coated tubes (Sarstedt, Nümbrecht, Germany). Samples were diluted 4-fold with saline for analysis on an Advia 2120 hematology analyzer (Siemens Healthcare, Erlangen, Germany). For in vivo study, peripheral blood $(50 \mu \mathrm{l})$ was collected every 2 to 4 days by tail bleeding up to day 15 and 21 , respectively.

Measurements of ROS and ATP levels. For measurement of complete ROS, BMC were incubated with $50 \mu \mathrm{M}$ 2',7'-dichlorofluorescein diacetate ((DCFH-DA); Sigma, Taufkirchen, Germany) for $30 \mathrm{~min}$. Fluorescence of $5 \times 10^{4}$ cells was measured using a Glomax ${ }^{\circledR}$ microplate reader (Promega, Mannheim, Germany) at a wavelength of $485 \mathrm{~nm}$.

To investigate mitochondrial superoxide, cells were stained with MitoSOX $^{\mathrm{TM}}$ (Invitrogen, Darmstadt, Germany) and Annexin-V-APC (Beckton Dickinson, Heidelberg, Germany) to exclude apoptotic cells from measurement. Cells were analyzed with a FACSCalibur (Beckton Dickinson, Heidelberg, Germany) and the proportion of MitoSOX ${ }^{\mathrm{TM}}$ positive and Annexin-V-APC-negative cells was counted.

Intracellular ATP levels of BMC were analyzed using the ATP Bioluminescence Assay Kit HS II (Roche, Mannheim, Germany). In brief, luminescence of cell lysates of $5 \times 10^{4}$ cells mixed with luciferase reagent was measured with a Glomax ${ }^{\circledR}$ reader. By using a standard serial dilution of ATP, the concentration in cell lysates could be determined.

Immunophenotyping. To evaluate the amount of the different subpopulations in the bone marrow, cells were stained with fluorescent antibodies (all obtained from Beckton Dickinson, Heidelberg, Germany) against different surface antigens: lymphocytes (IgG2 anti-CD3e phycoerythrin (PE) (Cat.-No. 553240), IgG2a anti-CD45R fluorescein isothiocyanate (FITC) (Cat. No. 553087)); erythroid cells (IgG2b anti-Ter-119 allophycocyanin (APC) (Cat.-No. 557909), IgG1 anti-CD71 PE (Cat.-No. 553267)); stem cells (IgG2b anti-c-kit PE (Cat.-No. 553355), IgG2a anti-Sca- 

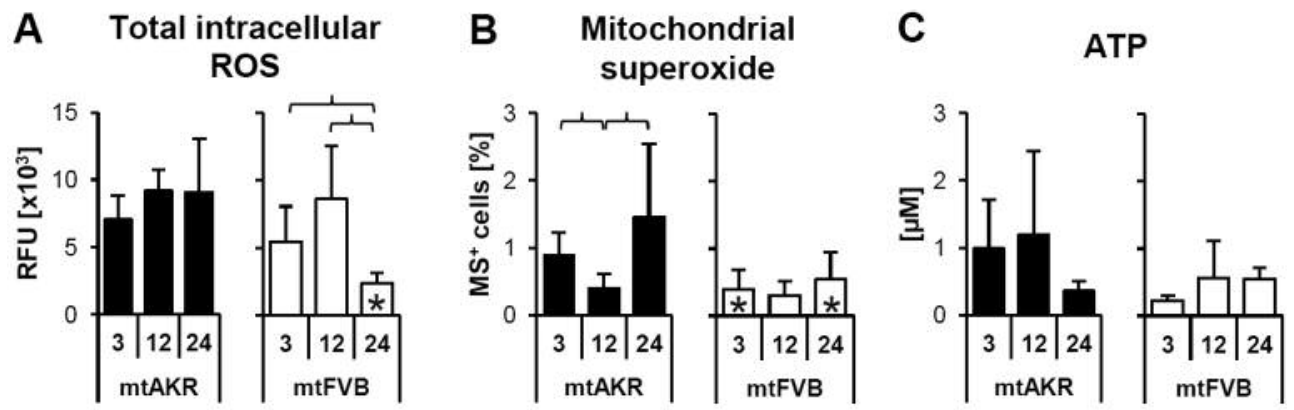

Figure 1. Polymorphism nt7778 G/T correlated with varying reactive oxygen species (ROS) and adenosine triphosphate (ATP) levels. (A) Total intracellular ROS in bone marrow cells was determined by staining with 2',7'-dichlorofluorescein diacetate (DCFH-DA) and measuring the relative fluorescence units (RFU). ROS levels are displayed for the three analyzed aging stages of both conplastic strains. (B) Bone marrow cells containing mitochondrial superoxide were stained with MitoSOX ${ }^{T M}$ and analyzed by flow cytometry. Additionally, the cells were stained with Annexin-V-APC to exclude apoptotic cells with unspecific signals. (C) ATP levels were evaluated by adding luciferase to cell lysates and measuring luminescence. *Significant difference versus mtAKR strain at same aging stage. Brackets indicate significant changes within one strain during aging. Six or more mice were investigated for each strain and aging stage.

1 PE- cyanine 7 (Cy7) (Cat.-No. 558162), lineage cocktail APC (Cat.-No. 558074), IgG2a anti-CD34 FITC (Cat.-No. 560238)) and corresponding isotype controls (hamster-IgG2 PE (Cat.-No. 550085), iso-lineage cocktail APC (Cat.-No. 558074), rat-IgG2a FITC (Cat.-No. 553929), rat-IgG2a PE-Cy7 (Cat.-No. 557855), ratIgG2b PE (Cat.-No. 556925), rat-IgG1 PE (Cat.-No. 554685), ratIgG2a FITC (Cat.-No. 553929)). Stained cells were analyzed with FACSCalibur (Beckton Dickinson). Total nucleated cells (TNC) were gated and the amount of cells positive for the distinct antigens was evaluated.

In vivo studies. The reconstitution capacity of the hematopoietic system was investigated by cytostatic stress induction. Animals were kept in a specific pathogen-free environment. After initial blood collection, animals were injected intraperitoneally with $150 \mathrm{mg} / \mathrm{kg}$ of body weight 5-fluorouracil (5-FU). The corresponding control group of each strain was treated with an equal volume of saline. For blood counts, whole blood $(50 \mu \mathrm{l})$ was taken every 2 to 4 days by tail bleeding up to days 15 and 21, respectively. After initially 21 days, bone marrow was isolated and investigated as described above. Later, bone marrow was analyzed at day 15 (12-month-old animals: $n=5,3$-month-old animals: mtAKR: $n=3$; mtFVB: $n=4$ ). Furthermore, spleen weight was measured. Data of the 5-FU-treated groups were analyzed in relation to the corresponding control group by calculating ratios. Study groups consisted of 10 mice.

Statistical analysis. Results within each experiment were described using mean and standard deviation. Significance between strains was calculated using Mann-Whitney $U$-test (SPSS Software, Version 22; IBM, Ehningen, Germany). A $p$-value $<0.05$ was considered to be significant.

\section{Results}

Polymorphism nt7778 G/T correlated with changes in ROS and ATP levels in aged mice. The ROS levels were measured by two different methods. Total cellular ROS was determined by DCFH-DA staining (Figure 1A) and the cells containing mitochondrial superoxide were analyzed by using MitoSOX $^{\mathrm{TM}}$ (Figure 1B). While control mtAKR animals showed a slight increase of intracellular ROS from 3 to 24 months $\left(7.1 \pm 1.7 \times 10^{3}\right.$ relative fluorescence units $(\mathrm{RFU})$ to $\left.9.0 \pm 4.0 \times 10^{3} \mathrm{RFU} ; p=0.628\right)$, the $\mathrm{mtFVB}$ strain displayed a significant decrease $\left(5.5 \pm 2.6 \times 10^{3}\right.$ RFU to $2.4 \pm 0.7 \times 10^{3} \mathrm{RFU}$; $p=0.037)$ over time. In comparison to the mtAKR strain, intracellular ROS levels at 24 months were significantly lower in mtFVB strain $(p<0.001)$.

Concerning mitochondrial superoxide, the mtAKR strain demonstrated an non-significant increase from $0.9 \pm 0.4 \%$ of MitoSOX ${ }^{\mathrm{TM}}$-positive cells in bone marrow at 3 months to $1.5 \pm 1.1 \%$ at 24 months $(p=0.414)$. The mtFVB strain showed almost stable levels with $0.4 \pm 0.3 \%$ in young animals and $0.5 \pm 0.4 \%$ in advanced-aged mice $(p=0.323)$. The superoxide levels at 3 and 24 months were significantly lower in mtFVB strain compared to mtAKR $(p=0.015$ and $p=0.030$, respectively). ATP levels changed in significantly

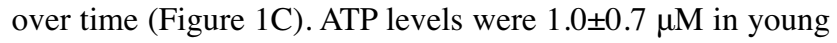
animals and $0.4 \pm 0.1 \mu \mathrm{M}$ in mtAKR mice of advanced age (Figure $1 \mathrm{C} ; p=0.867)$. In comparison, the $\mathrm{mtFVB}$ strain displayed levels from $0.2 \pm 0.1 \mu \mathrm{M}$ to $0.5 \pm 0.2 \mu \mathrm{M}(p=0.216)$.

In summary, the mtFVB strain displayed significantly lower levels of ROS and mitochondrial superoxide in elderly animals, while ATP levels were unaffected.

Polymorphism nt7778 G/T correlated with differing composition of the bone marrow during aging. The proportions of cellular subpopulations in the bone marrow changed during aging in both strains (Figure 2). The LSK cells were defined as lineage, Sca- $1^{+}$and $\mathrm{c}-\mathrm{kit}^{+}$. The percentage of LSK cells within the lineage- cells increased not significantly from 3 to 24 months (mtAKR: $2.1 \pm 0.7$ to $2.8 \pm 1.1 \% ; p=0.345$; mtFVB: $2.1 \pm 1.1$ to $3.5 \pm 1.6 \% ; p=0.106)$. However, the 
A
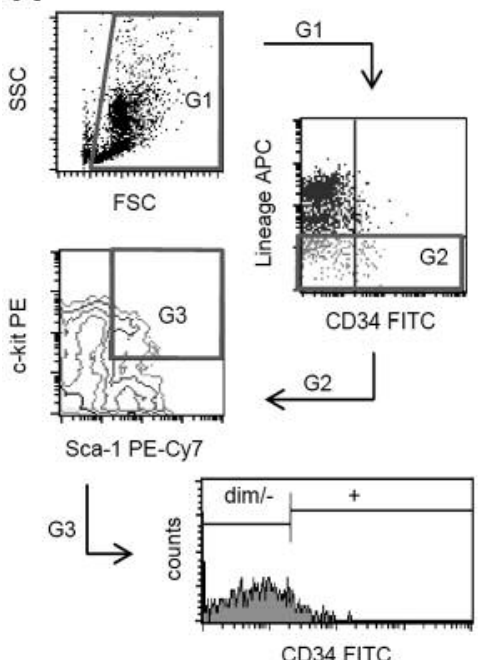

B
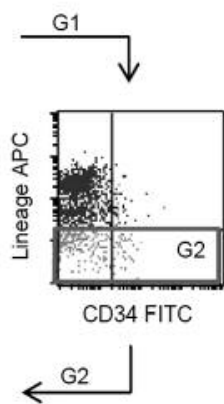
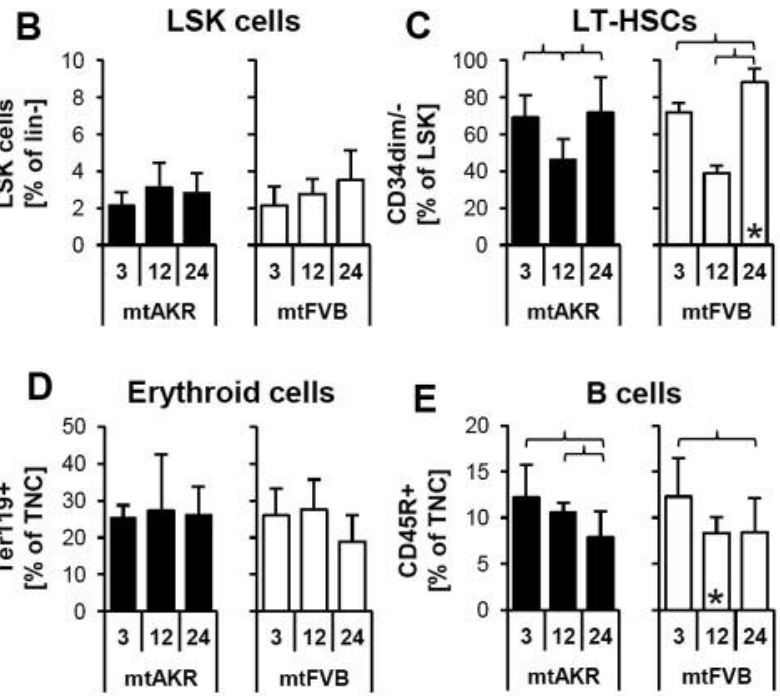

B cells

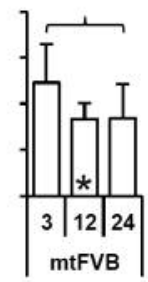

Figure 2. Polymorphism nt7778 G/T impacted the composition of bone marrow subpopulations. (A) Determination of LSK and LT-HSCs is shown as an example. Of the total nucleated cells ((TNC); G1), those negative for lineage commitment were determined (G2). Of these, cells positive for Sca-1 and c-kit were gated (G3; LSK cells shown in B). Subsequently, the proportion of cells with a diminished or no expression for CD34 was identified (LT-HSCs shown in C). Further, the amount of Ter119+ (erythroide cells $-D)$ and CD45R ${ }^{+}(B$ cells $-E)$ cells, respectively, was determined. *Significant difference versus mtAKR strain at same aging stage. Brackets indicate significant changes within one strain during aging. Six or more mice were investigated for each strain and aging stage.

proportion of $\mathrm{CD} 34^{\mathrm{dim} /-}$ cells in LSK pool increased significantly from 3 to 24 months in the mtFVB strain (72.0 \pm 4.8 to $88.2 \pm 7.5 \% ; p<0.001)$ and showed significantly higher levels compared to mtAKR mice at 24 months $(71.8 \pm 19.2 \% ; p=0.010)$. Both, the mtAKR, as well as the mtFVB strain, demonstrated a marginal increase of erythroid cells during aging $(25.2 \pm 3.5$ to $26.0 \pm 7.8 \%$ of TNC; $p=0.189$ (mtAKR); (25.9 \pm 7.3 to $18.9 \pm 7.2 \% ; p=0.149$ (mtFVB)).

Both strains exhibited a similar composition of lymphoid subpopulations. A slight increase in T cell numbers (data not shown) was detected, while B cells decreased in both strains during aging. Noteworthy, the mtFVB strain revealed an earlier decrease of $B$ cells in mid-aged mice with a significant lower proportion of B cells $(8.4 \pm 1.7 \%$ of TNCs) compared to mtAKR strain $(10.6 \pm 1.1 \% ; p=0.013)$.

To sum-up, the bone marrow composition of 24-month-old animals from the mtFVB strain differs significantly from mtAKR strain in the proportion of CD $34^{\mathrm{dim} /-}$ cells. They also tended to have fewer erythroid cells $(p=0.129)$. In addition, $B$ cell populations diminished at an earlier time point in mtFVB strain compared to mtAKR strain during aging.

Polymorphism nt7778 G/T correlated with reduced hemoglobin content of erythrocytes. Blood count analysis is an important parameter to evaluate hematopoietic stem cell function. In young and mid-aged mice, both strains displayed very similar parameter patterns (Figure 3). Mice of advanced age, however, showed distinct differences. Twenty-four-month-old mice of the mtAKR strain tended to have more leukocytes (white blood cells (WBC)) compared to the mtFVB strain $\left(6.2 \pm 3.3 \times 10^{3} / \mu \mathrm{l} v s\right.$. $\left.4.2 \pm 2.4 \times 10^{3} / \mu \mathrm{l} ; p=0.098\right)$. Although the $\mathrm{mtFVB}$ strain displayed similar numbers of erythrocytes compared to the mtAKR samples, the hemoglobin levels (HGB) were slightly lower at 24 months compared to the control strain $(10.8 \pm 1.7 \mu \mathrm{g} / \mu \mathrm{l} v \mathrm{~s}$. $12.1 \pm 1.4 \mu \mathrm{g} / \mu \mathrm{l} ; p=0.060)$. The mean corpuscular hemoglobin $(\mathrm{MCH})$ exhibited significantly lower levels in $\mathrm{mtFVB}$ mice than in the mtAKR strain at 24 months $(12.2 \pm 1.4 v s .14 .1 \pm 1.7 \mathrm{pg} / \mathrm{cell}$; $p=0.003$ ). Levels of both HGB and $\mathrm{MCH}$ in mtFVB animals were below the murine standard (HGB: 11.8-14.9 $\mu \mathrm{g} / \mu \mathrm{l}$; $\mathrm{MCH}$ : 13.8-18.4 pg/cell). Furthermore, in 24-month-old animals, the proportion of neutrophils (the most abundant type of granulocytes of total WBCs) was marginally higher in $\mathrm{mtFVB}$ strain with $8.6 \pm 3.6 \%$ than in the mtAKR strain with $6.0 \pm 2.0 \%$ $(p=0.059)$. Lymphocyte levels were not significantly different between the strains.

In summary, older mtFVB animals displayed a more distinct age-related decrease of $\mathrm{HGB}$ and $\mathrm{MCH}$ compared to mtAKR.

Polymorphism nt7778 G/T correlated with altered restoration of hematopoietic system after cytostatic stress in vivo. Following treatment with 5-FU, both strains developed a pancytopenia with subsequent recovery of blood parameters. The results of the 5-FU-treated animals were 

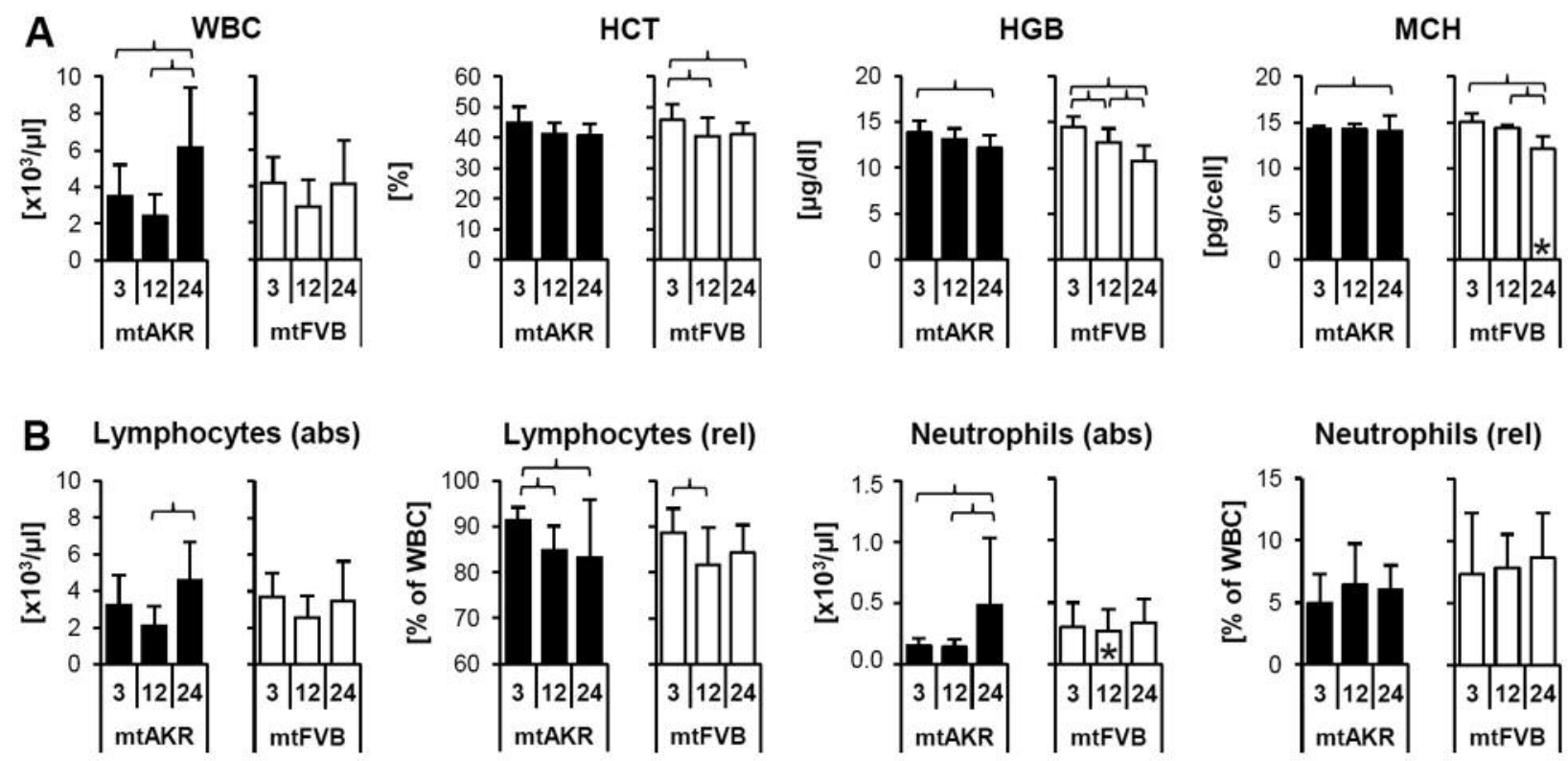

Figure 3. Polymorphism nt $7778 \mathrm{G} / \mathrm{T}$ affected hemoglobin content of erythrocytes. Blood parameters were measured with the Advia ${ }^{\circledR}$ device. (A) Results at the three investigated aging stages (3,12 and 24 months) are displayed for leukocytes (WBC), hematocrit (HCT), hemoglobin (HGB) and mean corpuscular hemoglobin $(\mathrm{MCH})$. (B) The composition of the leukocyte pool was investigated by determining the absolute (abs) and relative (rel) parameters of lymphocytes and neutrophils. *Significant difference versus mtAKR strain at same aging stage. Brackets indicate significant changes within one strain during aging. Seven or more mice were investigated for each strain and aging stage.

analyzed in relation to the data of the corresponding control group treated with saline solution by calculating ratios. Analysis of WBC, red blood count (RBC), hematocrit (HCT) and platelets (PLT) revealed similar patterns in both strains (Figure 4). Both strains showed a decrease of neutrophils after cytostatic stress with a subsequent rebound reaction (Figure 5). The mtAKR strain reached the nadir at day 6 at both aging stages with a ratio of $0.12 \pm 0.13$ in young mice and $0.07 \pm 0.05$ (Figure 5B) in older animals. Notably, young animals of the mtFVB strain also displayed the nadir at day 6 but with a higher ratio of $0.23 \pm 0.14(p=0.053)$. Mid-aged mice of mtFVB strain possessed more neutrophils at day 6 with a ratio of $0.20 \pm 0.14$; however, this difference did not reach statistical significance $(p=0.063)$. They presented their nadir at day 9 with a ratio of $0.13 \pm 0.10$. The rebound reaction of neutrophils in young animals from the $\mathrm{mtFVB}$ strain at day 15 was significantly higher with $2.51 \pm 1.23 \mathrm{vs}$. $1.21 \pm 0.53$ in mtAKR animals $(p=0.013)$. The latter displayed the restoration peak at day 18 with a ratio of $1.40 \pm 0.38$. Midaged mice of both strains showed a similar rebound reaction in neutrophils following cytostatic stress with a ratio of $2.65 \pm 1.66 \mathrm{in} \mathrm{mtAKR}$ and $2.72 \pm 0.69 \mathrm{in} \mathrm{mtFVB}$ strain at day 15 , respectively $(p=0.780)$.

The number of large unstained cells (LUC) was reduced after cytostatic application and demonstrated a rebound reaction also in both strains. Young mice of the mtFVB strain showed a lower nadir of LUC with $0.14 \pm 0.44$ at day 9 compared to the mtAKR strain with $0.33 \pm 0.43$ at day 2 . Interestingly, in young animals, the peak of the rebound reaction seemed higher $(2.15 \pm 1.02$ in $\mathrm{mtFVB}$ vs. $1.40 \pm 0.93$ in mtAKR, both at day 15); however, this difference was not significant $(p=0.779)$. Mid-aged mice displayed a more distinct depletion of LUC in the mtAKR strain with a ratio of $0.00 \pm 0.00$ at day 9 compared to $0.14 \pm 0.45$ in $\mathrm{mtFVB}$ animals at day 6. Again, the restoration peak tended to be higher in mtFVB than in mtAKR with a ratio of $8.40 \pm 7.85$ vs. $3.33 \pm 1.57(p=0.095)$.

The investigation of spleen and bone marrow at days 15 and 21 , respectively, revealed no significant differences between both strains when treated with 5-FU. At day 15, both strains displayed elevated levels of LSK cells and a splenomegaly, which was slightly more distinct in mtFVB strain (Figure 6). Other bone marrow subpopulations exhibited decreased levels compared to the corresponding control, while ROS and ATP levels did not show distinct deviations from control group (Figure 6). Analysis at day 21, after 5-FU treatment, revealed a normal distribution of subpopulations and almost normal spleen weight in both strains (data not shown).

Summarizing, the mtFVB strain displayed a similar regeneration of blood count after 5-FU treatment, except neutrophils and LUC, compared to mtAKR. Young animals 

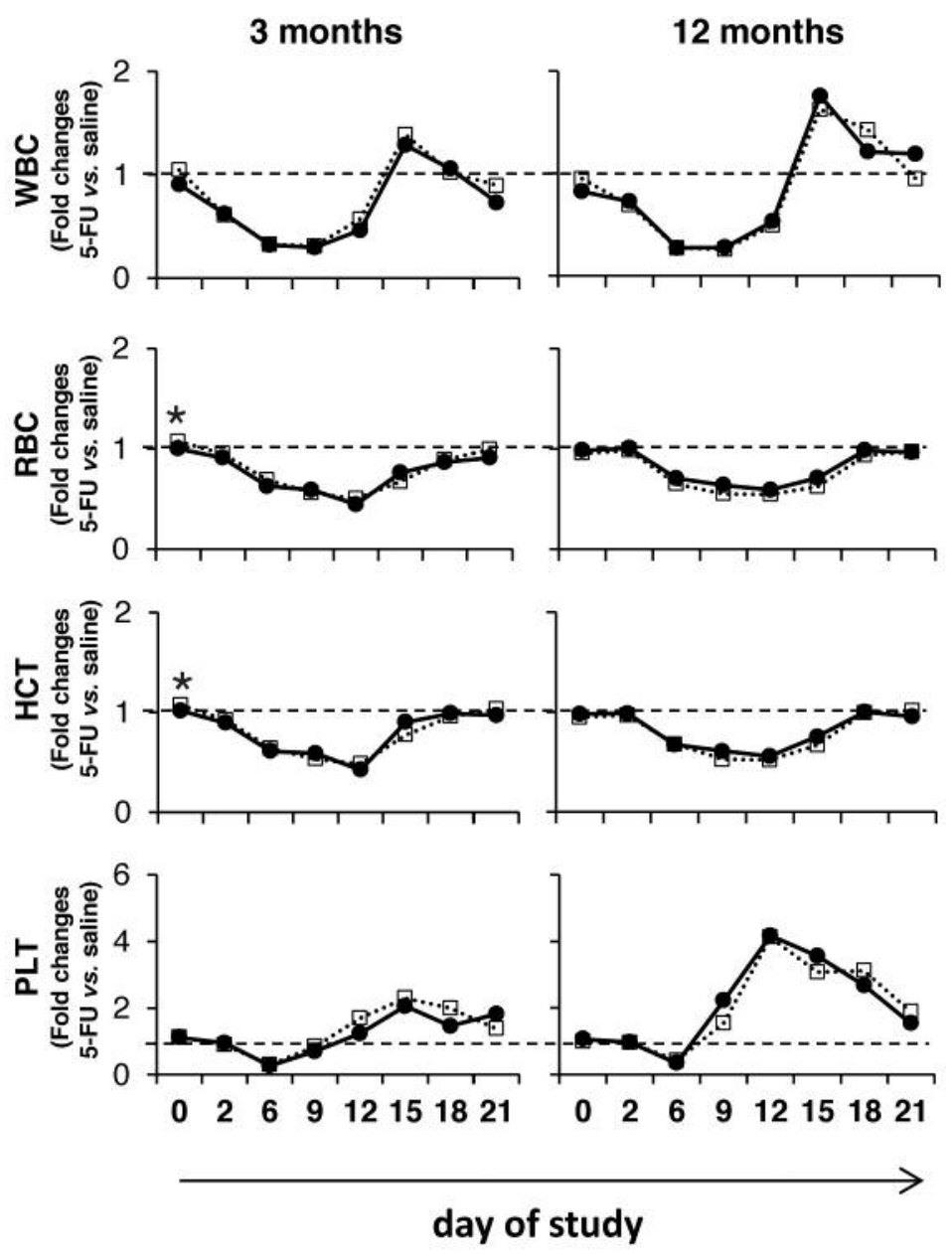

\section{$\multimap \quad$ mtAKR $\quad \ldots . \cdots \cdots \cdot m$ mtFVB}

Figure 4. Depletion and restoration of further blood parameters following cytostatic stress. Animals were injected with 5-fluorouracil (5-FU) (150 mg/kg of body weight) at day 0. Every 2-4 days, blood was taken by tail bleeding. Blood parameters were measured with the Advia ${ }^{\circledR}$ device. Data of 5-FU-treated animals are displayed in relation to the results of corresponding control group treated with saline. WBC, White blood cells; HCT, hematocrit; PLT, platelets; RBC, red blood cells.

of mtFVB exhibited a significantly stronger rebound regeneration of neutrophils than mtAKR. Further, 12-monthold mtFVB mice showed a trend to a stronger rebound reaction in LUC compared to mtAKR mice. Investigation of bone marrow at days 15 and 21 did not reveal considerable differences between the strains.

\section{Discussion}

Conplastic mouse strains represent a suitable model to evaluate the impact of a single mitochondrial polymorphism on organs, tissues and the whole organism. In this study, we investigated the influence of the nt7778 G/T polymorphism on the hematopoietic system. This polymorphism in ATP8 gene causes an amino acid exchange from aspartic acid to tyrosine in the corresponding protein of respiratory chain complex V. Here, ROS and ATP levels, as well as subpopulations in BMC, were analyzed during aging; also, blood count was investigated. In addition, an in vivo study evaluated the restoration capacity of the hematopoietic system after cytostatic stress.

The nt7778 G/T polymorphism correlated with changes in ROS levels in animals of advanced age. The mtFVB strain, harboring the amino acid exchanging polymorphism, showed a more distinct but not a significantly different increase of LSK cells from young to advanced-aged animals compared to the reference mtAKR. While aging HSCs were shown to increase with time $(2,25)$, it was indicated that elevated ROS 

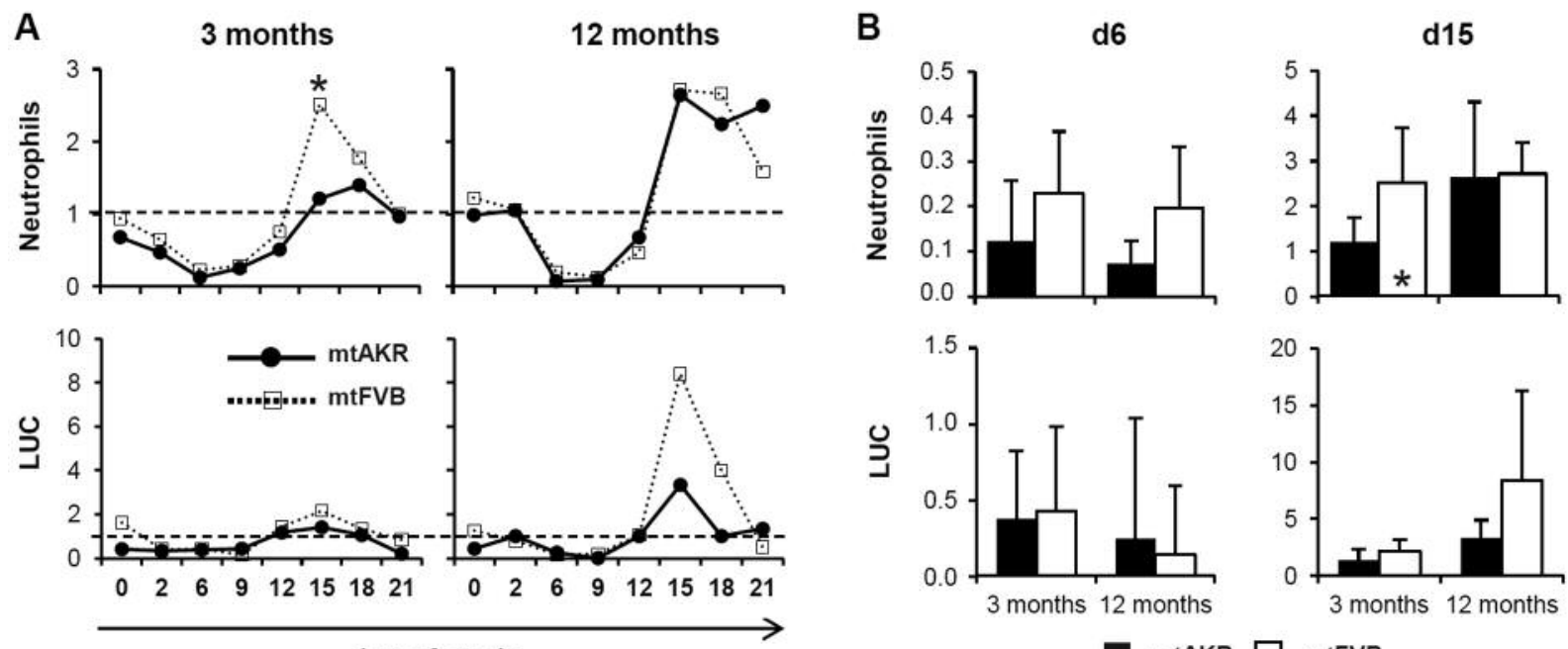

day of study

Figure 5. Polymorphism nt7778 G/T impacted restoration of hematopoietic system after cytostatic stress in vivo. Animals were injected intraperitoneally with 5-fluorouracil (5-FU) $(150 \mathrm{mg} / \mathrm{kg}$ of body weight) at day 0. Every 2-4 days, blood was taken by tail bleeding. Blood parameters were measured with Advia ${ }^{\circledR}$. Data of 5-FU-treated animals are displayed in relation to the results of the corresponding control group treated with saline. (A) Development of neutrophils and large unstained cells (LUC) after 5-FU treatment. (B) Neutrophils and LUCs are directly compared at days 6 and 15. *Significant difference with $p<0.05$.

levels in C57BL/6 mice led to an attenuated HSC maintenance and repopulation capacity (13). Furthermore, a more quiescent behavior of HSCs with higher maintenance capacity and less cycling was linked to low intracellular ROS levels (26). Here, mtFVB strain displayed a significantly higher proportion of CD34 ${ }^{\mathrm{dim} /-}$ cells in LSK pool. The lack of CD34 was shown to be characteristic for LT-HSCs in mice (27). The observation of lower ROS levels in old mtFVB mice with concomitantly higher proportion of CD34 $\mathrm{dim} /-$ cells in a larger LSK pool is in line with the already described correlation between low ROS and higher maintenance capacity of HSCs (26).

Interestingly, the investigation of pancreatic and hepatic cells of the mtFVB strain by other groups revealed enhanced ROS levels compared to controls. Pancreatic islet cells in 3-month-old mtFVB mice showed an increased basal production of ROS compared to mtAKR strain. At the same time a pancreatic $\beta$ cell dysfunction, as well as an altered mitochondrial morphology in spleen cells, could be demonstrated (21). Pancreatic acini of 12-month-old mtFVB mice, by trend, had also enhanced ROS levels in comparison to mtAKR mice (20). However, in a corresponding in vivo study, no aggravation of acute pancreatitis in mtFVB strain was observed. The induction of an acute endotoxemic liver failure revealed a survival advantage of mtFVB strain, although here oxidative stress was also enhanced compared to the background strain C57BL/6J (22).
Yu et al. also investigated the nt7778 G/T polymorphism but with different background strains that were all associated with autoimmune diseases. They reported a higher ROS production in spleen cells but no impaired mitochondrial respiration in 3- to 6-month-old animals (19). These findings differ from ours as we observed lower levels of ROS in BMC in the mtFVB strain. Therefore, the data suggest that murine tissues are differently impacted by the polymorphism concerning the formation of ROS. This could be due to the fact that the activation of distinct signaling pathways varies in different tissues, particularly under the influence of additional challenges, such as autoimmune stress. Given the participation of ROS in signaling, a varying ROS production, based upon tissue-specific pathway activation, appears plausible. Furthermore, different cell types were shown to posses varying activation of the respiratory chain with concomitantly differing ROS production. LT-HSCs, for example, were shown to cover their energy requirements by glycolysis instead of oxidative phosphorylation (28).

At the different aging stages studied here, complete intracellular ROS and mitochondrial superoxide did not change uniformly. Differences in complete ROS and superoxide can be explained by elevated degradation of superoxide to $\mathrm{H}_{2} \mathrm{O}_{2}$ by superoxide dismutases (SODs). Additionally, there are several enzymes in the peroxisome, as acyl-CoA oxidases, producing directly $\mathrm{H}_{2} \mathrm{O}_{2}$ independently from superoxide (29). 

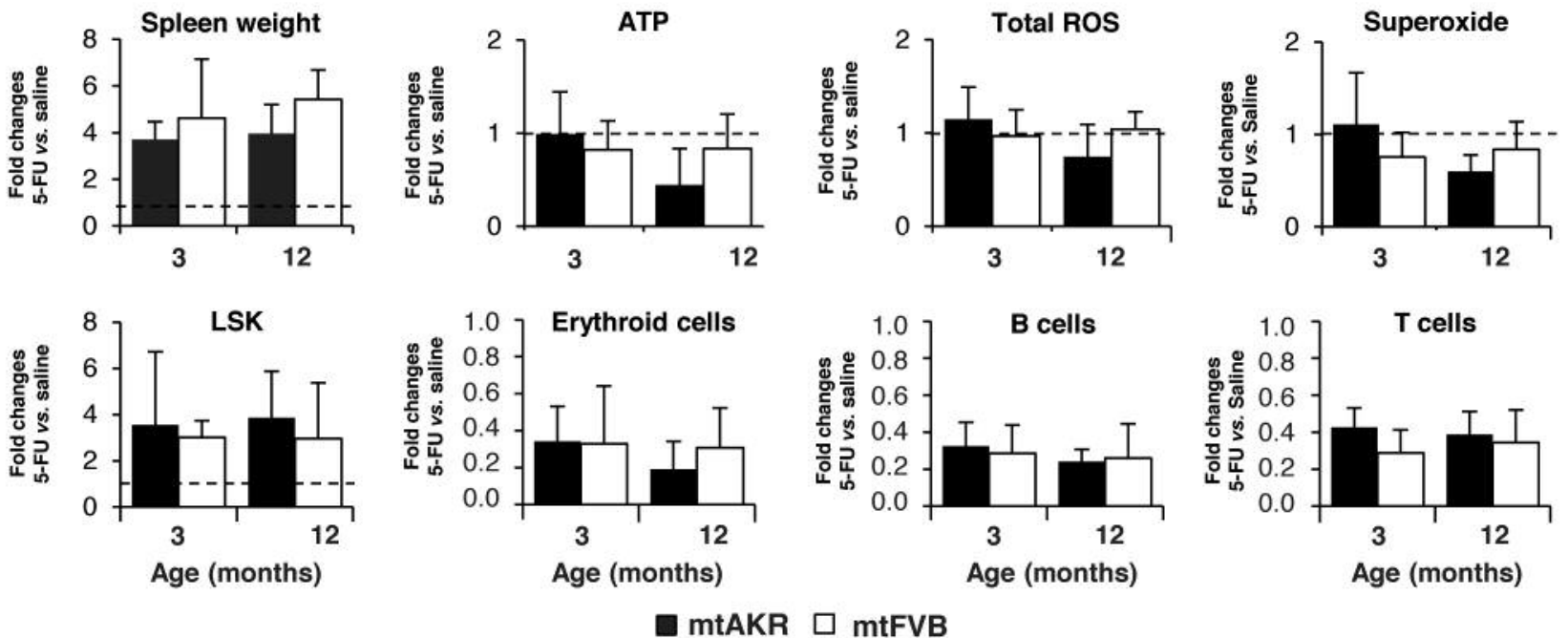

Figure 6. Spleen weight, reactive oxygen species (ROS) and adenosine triphosphate (ATP) levels and composition of bone marrow at day 15 post-5fluorouracil (5-FU) treatment. Spleen weight (ratio of spleen weight to body weight) of 5-FU-treated animals was determined and analyzed in relation to the weights of corresponding control groups treated with saline. Results for ROS and ATP levels, as well as composition of bone marrow cells, are shown at day 15. The results include data of 12-month-old animals ( $n=5$ for each strain) and 3-month-old mice (mtAKR: $n=3$; mtFVB: $n=4)$.

Numazawa et al. (30) pointed out that, in C57BL/6 mice, the administration of 5 -FU in hemotoxic doses led to an induction of heme oxygenase 1 , a known biomarker for oxidative stress, as well as a reduction of glutathion. Moreover, when keeping the glutathion pool constant by $\mathrm{N}$ acetylcysteine treatment, the 5-FU-induced hemotoxicity could be reduced, suggesting that ROS scavenging attenuated hemotoxic effects of 5-FU (30). These observations, combined with our findings of lower ROS in aged mice, lead to the assumption that if $\mathrm{mtFVB}$ mice would be protected from 5-FU-induced oxidative stress, the hemotoxicity should be less distinct. However, in our in vivo study, the hemotoxic effects on WBC, HCT and PLT were similar compared to the mtAKR strain. Solely, an impact on the peak recovery of neutrophils, as well as the LUC, was detectable. Basal total ROS levels showed similar values in 3- and 12-month-old mice of both strains and only a significant difference in animals of advanced age. In line with the observation of Ito et al. that enhanced ROS levels correlate with impaired HSC repopulation potential (13), the overall regeneration capacity, in our study, is similar in both strains in 3- and 12-month-old mice.

Interestingly, a higher, though marginal, increase of LUC proportion in peripheral blood in 12-month-old mtFVB mice at day 15 following 5-FU was detected, while the LSK proportion in the bone marrow was almost the same. The LUC fraction, described as large peroxidase-negative cells, also contains, apart from enlarged lymphocytes and virocytes, stem cells (31). LUC have been studied in the diagnosis and monitoring of acute leukemias and MDS (32). A recent study revealed a discriminating power of LUC in the investigation of acute promyelocytic leukemia (33). Furthermore, it was shown that an increase of LUC in peripheral blood during chemotherapy-induced neutropenia correlates with $\mathrm{CD} 34^{+}$cells and blasts in pre-nadir and postnadir phases (34). Moreover, an enlarged LUC population was also detected in other diseases like viral infections, chronic renal failure and malaria (35). Mice treated with 5FU, in our study, were kept in a low-germ environment and had no morbidities prior to treatment. Therefore, a possible explanation for the enlarged LUC population in the peripheral blood of mtFVB strain could be a higher stem cell mobilization. There are several studies investigating the influence of aging on stem cell mobilization. Xing et al. (36) performed a comparative assay in young and old mice investigating the influence of granulocyte-colony stimulating factor (G-CSF) on the mobilization of colony forming cells (CFC). A three-fold higher mobilization capacity of stem cells into the peripheral blood was observed in old animals, while no differences between young and aged mice were detectable in the bone marrow (36). Further, an in vitro assay showed a two-fold reduced adhesion to a cell monolayer in old compared to young hematopoietic progenitor cells (HPC) (36).

Another study reported a two-fold decrease in relative short-term homing capacities of old HSC when co-injected with young cells into irradiated recipient mice (37). Liang et al. also discovered an age-related decline in homing 
efficiency in murine HSC (38). In cancer patients, with standard dose chemotherapy, a highly significant positive correlation between the amount of LUC and CD34 $4^{+} / \mathrm{CD} 45^{+}$ cells in the peripheral blood was observed $(p<0.001)(34)$. This population corresponds to the hematopoietic stem and progenitor cells in humans (39). These results suggest that the enhanced LUC population in the peripheral blood of mtFVB mice after 5-FU treatment might be due to an aging hematopoietic system. Additionally, compared to mtAKR, a trend to less erythroid cells in the bone marrow of old mtFVB mice and a significantly earlier decrease in B cells were observed. This also points to an aging of the hematopoietic system, although the exact mechanisms have to be elucidated.

In summary, the amino acid exchanging mitochondrial nt7778 G/T polymorphism impacted first and foremost the ROS levels in murine BMC. While ROS levels decreased significantly with age, ATP levels were unaffected. Moreover, significantly lower hemoglobin content in erythrocytes was detectable in older mice. Stress induction by cytostatic administration revealed no homogenous marked differences between both strains investigated.

The underlying signaling cascades leading to the effects of the mtDNA variation remain to be investigated, as well as the different impact on various cell types and tissues concerning ROS levels.

\section{Conflicts of Interest}

There is no conflict of interest to declare.

\section{Acknowledgements}

The Authors would like to thank the animal attendants of the central animal facility of the University of Rostock for providing the facilities. The Authors thank Emma Woodham for the critical reading of the manuscript and helpful advice.

This work was funded by the German Federal Ministry of Education and Research (BMBF) as part of the GerontoSys initiative (ROSAge project), FKZ 0315892A.

\section{References}

1 Dykstra B and de Haan G: Hematopoietic stem cell aging and self-renewal. Cell Tissue Res 331: 91-101, 2008.

2 Moeller M, Hirose M, Mueller S, Roolf C, Baltrusch S, Ibrahim S, Junghanss C, Wolkenhauer O, Jaster R, Köhling R, Kunz M, Tiedge M, Schofield PN and Fuellen G: Inbred mouse strains reveal biomarkers that are pro-longevity, antilongevity or role switching. Aging Cell 13: 729-738, 2014.

3 Geiger H, Denkinger M and Schirmbeck R: Hematopoietic stem cell aging. Curr Opin Immunol 29: 86-92, 2014.

4 Rübe CE, Fricke A, Widmann $\mathrm{T}$ a, Fürst $\mathrm{T}$, Madry $\mathrm{H}$, Pfreundschuh $\mathrm{M}$ and Rübe $\mathrm{C}$ : Accumulation of DNA damage in hematopoietic stem and progenitor cells during human aging. PLoS One 6: e17487, 2011.
5 Chung YJ, Robert C, Gough SM, Rassool F V and Aplan PD: Oxidative stress leads to increased mutation frequency in a murine model of myelodysplastic syndrome. Leuk Res 38: 95-102, 2014.

6 Inoue S-I, Noda S, Kashima K, Nakada K, Hayashi J-I and Miyoshi $\mathrm{H}$ : Mitochondrial respiration defects modulate differentiation but not proliferation of hematopoietic stem and progenitor cells. FEBS Lett 584: 3402-3409, 2010.

7 Chen ML, Logan TD, Hochberg ML, Shelat SG, Yu X, Wilding GE, Tan W, Kujoth GC, Prolla T, Selak M, Kundu M, Carroll M and Thompson JE: Erythroid dysplasia, megaloblastic anemia, and impaired lymphopoiesis arising from mitochondrial dysfunction. Blood 114: 4045-4053, 2009.

8 Ito K, Hirao A, Arai F, Matsuoka S, Takubo K, Hamaguchi I, Nomiyama K, Hosokawa K, Sakurada K, Nakagata N, Ikeda Y, Mak TW and Suda T: Regulation of oxidative stress by ATM is required for self-renewal of haematopoietic stem cells. Nature 431: 997-1002, 2004.

9 Larsson N-G: Somatic mitochondrial DNA mutations in mammalian aging. Annu Rev Biochem 79: 683-706, 2010.

10 Park S, You X and Imlay J: Substantial DNA damage from submicromolar intracellular hydrogen peroxide detected in $\mathrm{Hpx}$ mutants of Escherichia coli. Proc Natl Acad Sci USA 102: 93179322, 2005.

11 Avery SV: Molecular targets of oxidative stress. Biochem J 434: 201-210, 2011.

12 Freinbichler W, Colivicchi M, Stefanini C, Bianchi L, Ballini C, Misini B, Weinberger P, Linert W, Varešlija D, Tipton KF and Della Corte L: Highly reactive oxygen species: Detection, formation, and possible functions. Cell Mol Life Sci 68: 2067-2079, 2011.

13 Ito K, Hirao A, Arai F, Takubo K, Matsuoka S, Miyamoto K, Ohmura M, Naka K, Hosokawa K, Ikeda Y and Suda T: Reactive oxygen species act through p38 MAPK to limit the lifespan of hematopoietic stem cells. Nat Med 12: 446-451, 2006.

14 Liu S-L, Lin X, Shi D-Y, Cheng J, Wu C-Q and Zhang Y-D: Reactive oxygen species stimulated human hepatoma cell proliferation via cross-talk between PI3-K/PKB and JNK signaling pathways. Arch Biochem Biophys 406: 173-182, 2002.

15 Martindale JL and Holbrook NJ: Cellular response to oxidative stress: signaling for suicide and survival. J Cell Physiol 192: 1$15,2002$.

16 Caporossi D, Ciafrè SA, Pittaluga M, Savini I and Farace MG: Cellular responses to $\mathrm{H}(2) \mathrm{O}(2)$ and bleomycin-induced oxidative stress in L6C5 rat myoblasts. Free Radic Biol Med 35: 1355$1364,2003$.

17 Sigaud S, Evelson P and González-Flecha B: $\mathrm{H}_{2} \mathrm{O}_{2}$-induced proliferation of primary alveolar epithelial cells is mediated by MAP kinases. Antioxid Redox Signal 7: 6-13, 2005.

18 Sattler M, Winkler T, Verma S, Byrne CH, Shrikhande G, Salgia $\mathrm{R}$ and Griffin JD: Hematopoietic growth factors signal through the formation of reactive oxygen species. Blood 93: 2928-2935, 1999.

$19 \mathrm{Yu} \mathrm{X,} \mathrm{Wester-Rosenlöf} \mathrm{L,} \mathrm{Gimsa} \mathrm{U,} \mathrm{Holzhueter} \mathrm{S-A,} \mathrm{Marques} \mathrm{A,}$ Jonas L, Hagenow K, Kunz M, Nizze H, Tiedge M, Holmdahl R and Ibrahim SM: The mtDNA nt7778 G/T polymorphism affects autoimmune diseases and reproductive performance in the mouse. Hum Mol Genet 18: 4689-4698, 2009.

20 Müller S, Krüger B, Lange F, Bock CN, Nizze H, Glass Ä, Ibrahim SM and Jaster R: The mtDNA nt7778 G/T polymorphism augments formation of lymphocytic foci but does not aggravate cerulein-induced acute pancreatitis in mice. PLoS One 9: e102266, 2014. 
21 Weiss H, Wester-Rosenloef L, Koch C, Koch F, Baltrusch S, Tiedge $\mathrm{M}$ and Ibrahim S: The mitochondrial Atp8 mutation induces mitochondrial ROS generation, secretory dysfunction, and $\beta$-cell mass adaptation in conplastic B6-mtFVB mice. Endocrinology 153: 4666-4676, 2012.

22 Eipel C, Hildebrandt A, Scholz B, Schyschka L, Minor T, Kreikemeyer B, Ibrahim SM and Vollmar B: Mutation of mitochondrial ATP8 gene improves hepatic energy status in a murine model of acute endotoxemic liver failure. Life Sci 88: 343-349, 2011.

23 Gimsa U, Kanitz E, Otten W and Ibrahim SM: Behavior and stress reactivity in mouse strains with mitochondrial DNA variations. Ann NY Acad Sci 1153: 131-138, 2009.

24 Yu X, Gimsa U, Wester-Rosenlöf L, Kanitz E, Otten W, Kunz $M$ and Ibrahim SM: Dissecting the effects of mtDNA variations on complex traits using mouse conplastic strains. Genome Res 19: 159-165, 2009.

25 Beerman I, Maloney WJ, Weissmann IL and Rossi DJ: Stem cells and the aging hematopoietic system. Curr Opin Immunol 22: 500-506, 2010.

26 Jang Y-Y and Sharkis SJ: A low level of reactive oxygen species selects for primitive hematopoietic stem cells that may reside in the low-oxygenic niche. Blood 110: 3056-3063, 2007.

27 Osawa M, Hanada K, Hamada H and Nakauchi H: Long-term lymphohematopoietic reconstitution by a single CD34-low/ negative hematopoietic stem cell. Science 273: 242-245, 1996.

28 Simsek T, Kocabas F, Zheng J, Deberardinis RJ, Mahmoud AI, Olson EN, Schneider JW, Zhang CC and Sadek HA: The distinct metabolic profile of hematopoietic stem cells reflects their location in a hypoxic niche. Cell Stem Cell 7: 380-390, 2010

29 Schrader M and Fahimi HD: Peroxisomes and oxidative stress. Biochim Biophys Acta 1763: 1755-1766, 2006.

30 Numazawa S, Sugihara K, Miyake S, Tomiyama H, Hida A, Hatsuno M, Yamamoto M and Yoshida T: Possible involvement of oxidative stress in 5-fluorouracil-mediated myelosuppression in mice. Basic Clin Pharmacol Toxicol 108: 40-45, 2011.

31 Thirup P: LUC, what is that? Large unstained cells. Clin Chem 45: 1100,1999
32 Bononi A, Lanza F, Ferrari L, Gusella M, Gilli G, Abbasciano V, Campioni D, Russo A, Menon D, Albertini F, Stievano L, Barile C, Crepaldi G, Toso S, Ferrazzi E and Pasini F: Predictive value of hematological and phenotypical parameters on postchemotherapy leukocyte recovery. Cytometry B Clin Cytom 76: 328-333, 2009.

33 Jang MJ, Choi HW, Lee SY, Lee OJ, Kim HR, Shin JH and Suh SP: Application of bone marrow samples for discrimination of acute promyelocytic leukemia from other types of acute leukemia using the routine automated hematology analyzer. 531-540, 2014.

34 Bononi A, Lanza F, Dabusti M, Gusella M, Gilli G, Menon D, Toso S, Crepaldi G, Marenda B, Abbasciano V and Ferrazzi E: Increased myeloperoxidase index and large unstained cell values can predict the neutropenia phase of cancer patients treated with standard dose chemotherapy. Commun Clin Cytom 46: 92-97, 2001.

35 Nixon DF, Parsons J and Eglin RP: Routine full blood counts as indicators of acute viral infections. J Clin Pathol 40: 673-675, 1987.

36 Xing Z, Ryan M, Daria D, Nattamai KJ, Van Zant G, Wang L, Zheng $\mathrm{Y}$ and Geiger $\mathrm{H}$ : Increased hematopoietic stem cell mobilization in aged mice. Blood 108: 2190-2197, 2006.

37 Dykstra B, Olthof S, Schreuder J, Ritsema M and de Haan G: Clonal analysis reveals multiple functional defects of aged murine hematopoietic stem cells. J Exp Med 208: 2691-2703, 2011.

38 Liang Y, Van Zant G and Szilvassy SJ: Effects of aging on the homing and engraftment of murine hematopoietic stem and progenitor cells. Blood 106: 1479-1487, 2005.

39 Rieger M and Schroeder T: Hematopoiesis. Cold Spring Harb Perspect Biol 4: 1-17, 2012.
Received August 31, 2016

Revised September 15, 2016

Accepted September 16, 2016 